\title{
Cancers of unknown primary origin (CUP) are characterized by chromosomal instability (CIN) compared to metastasis of know origin
}

\author{
Jonas Vikeså ${ }^{1 \dagger}$, Anne Kirstine H Møller ${ }^{2 \dagger}$, Bogumil Kaczkowski ${ }^{3 \dagger}$, Rehannah Borup ${ }^{1 \dagger}$, Ole Winther ${ }^{2,5}$, \\ Ricardo Henao ${ }^{2,5}$, Anders Krogh ${ }^{2}$, Katharina Perell ${ }^{2}$, Flemming Jensen ${ }^{4}$, Gedske Daugaard ${ }^{2 \dagger}$ and Finn C Nielsen ${ }^{1 * \dagger}$
}

\begin{abstract}
Background: Cancers of unknown primary (CUPs) constitute 5\% of all cancers. The tumors have an aggressive biological and clinical behavior. The aim of the present study has been to uncover whether CUPs exhibit distinct molecular features compared to metastases of known origin.

Methods: Employing genome wide transcriptome analysis, Linear Discriminant Analysis (LDA) and Quadratic Discriminant Analysis (QDA), we defined the putative origins of a large series of CUP and how closely related a particular CUP was to corresponding metastases of known origin. LDA predictions were subsequently used to define a universal CUP core set of differentially expressed genes, that by means of gene set enrichment analysis was exploited to depict molecular pathways characterizing CUP.
\end{abstract}

Results: The analyses show that CUPs are distinct from metastases of known origin. CUPs exhibit inconsistent expression of conventional cancer biomarkers and QDA derived outlier scores show that CUPs are more distantly related to their primary tumor class than corresponding metastases of known origin. Gene set enrichment analysis showed that CUPs display increased expression of genes involved in DNA damage repair and mRNA signatures of chromosome instability (CIN), indicating that CUPs are chromosome unstable compared to metastases of known origin.

Conclusions: CIN may account for the uncommon clinical presentation, chemoresistance and poor outcome in patients with CUP and warrant selective diagnostic strategies and treatment.

Keywords: Carcinoma of unknown primary, Chromosome instability

\section{Background}

Cancers of unknown primary origin (CUPs) are a heterogeneous group of cancers with variable clinical and histological features for which no primary site of the tumor can be identified despite an extensive diagnostic work-up [1,2]. CUPs accounts for 3-5\% of all cancer diagnoses and about $85 \%$ of the patients have a very poor prognosis [3]. Although a primary tumor cannot be identified in about two-thirds of the cases, CUPs are generally considered to represent metastases. The elusive origin may partly be related to limitations in our

\footnotetext{
* Correspondence: fcn@rh.dk

${ }^{\dagger}$ Equal contributors

'Center for Genomic Medicine, Rigshospitalet, University of Copenhagen, Blegdamsvej 9, DK-2100 Copenhagen $\varnothing$, Denmark

Full list of author information is available at the end of the article
}

diagnostic procedures, but it may also indicate that CUPs exhibit distinct biological features compared to metastasis of known origin [4].

The prevalent model of metastasis is that cells from a primary tumor invade the local environment and spread to distant locations. Metastases may derive from more or less differentiated cancer cells at different stages of tumor growth and this may provide a substantial heterogeneity in the clinical presentation and nature of metastases. Although micrometastases are enriched in cells expressing stem cell markers, macrometastases share many similarities to the primary tumor, so newly settled cancer stem cells not only self-renew, but also foster differentiated colonies of cancer cells [5]. Because metastases retain some of the characteristics of the primary 
cancer, transcriptome signatures have been employed to depict the origin of CUPs.

It is currently unknown if CUPs exhibit particular genetic and phenotypic characteristics compared to metastases of known origin. The challenge in addressing this problem is obviously that CUPs per definition are of unknown origin. To circumvent this problem, we generated a molecular signature that could classify a wide number of known primary tumor classes and their metastases with high accuracy.

We used the expression signature to classify the CUPs and included a Quadratic Discrimination Analysis (QDA) to generate an outlier score depicting how closely related a particular sample is to the different kinds of primary tumors. Subsequently, we used the LDA predicted classification to make a paired analysis comparing CUPs to their equivalent metastasis of known origin (MOKO), to define a CUP core set of differentially expressed genes that could provide leads to the molecular pathology of CUPs.

We demonstrate that CUPs exhibit a number of distinct molecular features that distinguish them from conventional metastasis. CUP gene expression signatures are more distantly related to their predicted primary tumor classes than signatures of metastases known origin, and they exhibit an inconsistent expression of conventional cancer biomarkers. CUPs are enriched in BRCA1, ATM and CHEK2 DNA damage and homologous recombination repair networks suggesting that CUPs are chromosome unstable and this was corroborated by the demonstration of signatures of chromosome instability (CIN) in CUPs. The results indicate that CUPs may warrant selective diagnostic and therapeutic strategies distinct from the current platinum based and organ specific therapy.

\section{Methods}

\section{Gene expression profiles for tumor classification}

Expression profiles of more than 2400 tumor samples were downloaded from the Gene Expression Omnibus (GEO) (http://www.ncbi.nlm.nih.gov/geo/) (Testset: GSE2109, GSE7307, GSE6004, GSE6764, GSE10135, GSE2328, GSE13471, GSE7392 and GSE12606) (Validationset: GSE2109, GSE3325, GSE5764, GSE5764, GSE5787, GSE7307, GSE7476, GSE7553, GSE10245, GSE11151, GSE14762, GSE15471, GSE17537, GSE19826, GSE19829, GSE20565) or generated from samples collected and processed at our own facility at Rigshospitalet (ArrayExpress, E-MTAB-3222). Finally thyroid samples were retrieved from ArrayExpress data base (accession E-MEXP-2442). The specific identifiers of the samples are depicted in Additional file 1: Table S1. The numbers refer to the GSM number in the GEO profile data base and the name (e.g. breast) refers to the biopsy tissue site. The material comprised 15 classes of carcinomas from thyroid, lung, stomach, colon/rectum, pancreas, bile duct/gallbladder, liver, kidney, urinary tract, prostate, breast, ovary, endometrium, cervix uteri, testis cancer and 1 group of malignant melanomas and finally a group with pooled normal tissue samples from various organs that was included in order to allow detection of samples without sufficient tumor tissue. The 16 tumor classes were selected to represent the most frequently identified primary tumor sites in CUP patients at autopsy, and primary tumors that are difficult to distinguish by IHC tools alone due to the lack of specific IHC markers (e.g. upper GI) and/or tumor dedifferentiation. Each tumor class contained the most common histological subtypes. Sample IDs are indicated in the enclosed Additional file 1: Table S1. The pathology descriptions were reviewed in order to group the samples into tumor classes and this ultimately resulted in a set of 1466 expression profiles from well-defined primary tumors (1299) and normal tissue (167) (Additional file 1: Table S1). The classifier was tested on an independent validation set including 641 tumor samples (391 primary tumors and 250 metastases) from all 16 tumor classes (Additional file 1: Table S1).

\section{CUP patients and samples}

CUP patients were consecutively enrolled between November 2004 and September 2010 for diagnostic workup and treatment. Newly diagnosed CUP patients were referred to the Department of Oncology (Rigshospitalet) for further diagnostic work-up and treatment. All patients had a biopsy-proven metastatic cancer and had undergone diagnostic work-up at the referral hospitals. At the Department of Oncology at Rigshospitalet further diagnostic work-up was performed including revision of biopsies by an experienced pathologist, new biopsies and further imaging procedures. A schematic representation of the CUP patients and the inclusion of samples are shown in Additional file 2: Figure S1. Patients were included when the diagnostic work-up, as recommended by the European Society of Medical Oncology (ESMO) [6], failed to identify a primary site of origin. At least two ultra-sonographyguided biopsies - one for histopathological work-up and one for gene expression profiling - were obtained from all patients. Patients, in whom a putative a primary tumor site eventually was identified in the diagnostic work-up period, were treated according to national guidelines whereas most CUP patients were offered platin/taxane-based regimens as first-line treatment. The study was approved by the Danish RegionH ethical committee and patients had given their written informed consent and have consented for publication and disclosure of clinical data.

\section{Microarray analysis and expression values}

Total RNA was isolated, labeled and hybridized as described [7]. Cell files were pre-processed using the Robust 
multi-chip average (RMA) method [8] and evaluated for quality parameters with the Simpleaffy functionality of the $R /$ Bioconductor packages. The data sets were filtered to exclude probe sets with Interquartile Range (IQR) below 0.8 .

\section{Tumor classification and outlier analysis}

Linear discriminant analysis (LDA) was used for classification as implemented in the $\mathrm{R}$ language. Briefly, in LDA the predictive probability of class $\mathrm{c}$ given input $\mathrm{x}$ is computed using Bayes' theorem $\mathrm{p}(\mathrm{c} \mid \mathrm{x})=\mathrm{p}(\mathrm{x} \mid \mathrm{c}) \mathrm{p}(\mathrm{c}) / \mathrm{p}(\mathrm{x})$, where $\mathrm{p}(\mathrm{x} \mid \mathrm{c})$ is a normal density specific for the class, $\mathrm{p}(\mathrm{c})$ the a priori probability of class $\mathrm{c}$ and $\mathrm{p}(\mathrm{x})=$ sum_c $\mathrm{p}(\mathrm{x} \mid \mathrm{c}) \mathrm{p}(\mathrm{c})$ the density of the input according to the model. Maximum likelihood is used to fit $\mathrm{p}(\mathrm{x} \mid \mathrm{c})$ and $\mathrm{p}(\mathrm{c}), \mathrm{c}=1, \ldots, 17$ on the training data. In order to construct a gene signature for our classifier we used leaveone-out cross validation (LOOCV), where for each split, feature selection by F-test were applied prior to LDA. A grid search over p-value cut-offs yielded the cut-off with the optimal LOOCV accuracy. The signature was eventually selected by an F-test using the optimal p-value cut-off on the full set of 1466 training samples, resulting in 428 probes (311 unique genes). The performance of this first (428 probe) classifier was then assessed using the independent 641 sample validation set. We merged the original training and validation set and used the found p-value cut-off (giving 641 probes) to generate a second classifier optimized for CUP prediction. The performance of this classifier was assessed using LOOCV. Finally, the LDA classifier was made sex-specific by setting the prior probabilities to zero for sex specific cancers (ovary, cervical and prostate) not occurring and in the sex in question renormalizing the remaining prior probabilities accordingly. A low model density $\mathrm{p}(\mathrm{x})$ implied that the input $\mathrm{x}$ was not similar to those in the training data. We therefore defined an outlier score $\mathrm{OS}=-\log \mathrm{p}(\mathrm{x})$ and calculated the OS for each sample in the LOOCV loop. We used QDA (individual covariance of normals) rather than LDA (shared covariance of normals) in this step.

\section{Gene set enrichment analysis}

A CUP core list of transcripts was defined by a paired analysis between CUP LDA predictions and corresponding metastasis of known origin. The pairing was done by making a linear model of the data by eliminating the difference between the groups as implemented in the Qlucore Omics Explorer $^{\text {rt }}$ software. Analysis of the CUP core lists (up and down) was performed using the Broad Institutes MSig "Compute overlaps for selected genes" function available on the homepage http://www.broadinstitute.org/gsea/ msigdb). Gene symbols in the CUP core lists were analyzed for enrichments of Gene Onthology (GO) genesets
(C5). CUP core lists were also analyzed for enrichments of gene sets in the $\mathrm{cu}$ rated gene set database $(\mathrm{C} 2)$. The $\mathrm{C} 2$ gene set collection is gathered from various online pathway databases, publications from PubMed and knowledge of domains experts (see homepage). A filter setting was added to both analyses to show only gene sets with FDR q-value below 0.01. GSEA on predefined gene sets were performed using the Broad Institute GSEA v2 software. The expression data matrix was preprocessed in the Qlucore Omics Explorer ${ }^{\mathrm{rm}}$ software and expression values were normalized within LDA predictions. The data set was analyzed employing 1000 permutations with all the default standard settings of the GSEA v2 software. Hierarchical cluster analysis was performed and visualized using the Qlucore Omics Explorer ${ }^{\text {re }}$ software. All hierarchical clusters are build using average linkage and heat map was generated based on mean $m=0$, variance 1 normalization.

\section{Results}

\section{CUP patients and tumor classification}

Sixty eight consecutive CUP patients were enrolled in the study, but since eleven samples did not meet the quality criteria the number of CUP samples ended at 57. The histological features of the 57 CUP that underwent expression profiling are summarized in Table 1. During the diagnostic work-up, a possible primary tumor site was eventually identified in 28 of the 57 patients (Additional file 2: Figure S1 and Table 1). Among these 18 samples were in accordance with diagnostic work-up or the Standard of Reference.

To examine if CUP exhibit particular genetic and phenotypic characteristics compared to metastases of known origin, that could warrant particular diagnostic procedures and treatment, we first generated a transcriptome-based signature that could classify 16 common tumor classes and predict the origins of CUP and metastases of known origin with high accuracy (Detailed in Additional file 1: Table S1). To allow detection of samples without sufficient tumor tissue, a group of normal tissues was also included. Since all CUP data were generated at our facility, we moreover examined a series of primary cancers and metastases from Rigshospitalet to exclude possible site- and batchspecific effects. The cross-validation accuracy during training of a 428 probe sets classifier was $92.2 \%$ (Additional file 3: Table S2) and the overall accuracy in the validation set was $90 \%$ and $83 \%$ for primary tumors and known metastases, respectively (Additional file 3: Table S2). The distribution of variables among the 16 tumor categories is depicted in the heat map (Figure 1). Since we suspected that the low accuracy in some of the classes, e.g. cholangiocarcinoma, was associated with the small number of samples in the training set, and because CUPs were 
Table 1 Prediction results in CUP patients

\begin{tabular}{|c|c|c|c|c|c|c|c|}
\hline ID & Sex & Biopsy site & Histology & Path Diag. & Stand of Ref & LDA Pred & Outlier score \\
\hline 14. & $F$ & LN neck & PDC & Lung & Lung (CD) & Lung & 975 \\
\hline 17. & $\mathrm{~F}$ & LN neck & Adenoc. & Lower Gl & Colon (RD) & Colon & 746 \\
\hline 22. & M & LN neck & Adenoc. & CUP & Stomach (RD) & Normal & 934 \\
\hline 23. & M & LN retro & PDC & CUP & Kidney (RD) & Kidney & 1085 \\
\hline 28. & $\mathrm{~F}$ & Peritoneum & PDA & Ovary & Ovary (RD) & Ovary & 810 \\
\hline 31. & $\mathrm{~F}$ & LN neck & PDA & CUP & Lung (CD) & Stomach & 985 \\
\hline 34 & M & Skin & PDA & Lung & Lung (RD) & Lung & 898 \\
\hline 39. & M & Liver & Adenoc. & CUP & Pancreas (CD) & Pancreas & 1097 \\
\hline 40. & $\mathrm{~F}$ & Liver & Adenoc. & Colon & Colon (RD) & Colon & 729 \\
\hline 44. & $\mathrm{~F}$ & Kidney & Carc. & CUP & Bladder (RD) & Bladder & 1286 \\
\hline 49. & M & LN neck & PDA & Kidney & Kidney (RD) & Kidney & 1223 \\
\hline 51. & $\mathrm{~F}$ & LN pelvis & SCC & CUP-SCC & Cervical (RD) & Cervix & 828 \\
\hline 52. & M & Liver & PDA & CUP & $\mathrm{CCC}(\mathrm{RD})$ & $\mathrm{CCC}$ & 923 \\
\hline 53. & M & Liver & Adenoc. & Lung & Lung (RD) & Lung & 1047 \\
\hline 57. & M & Liver & Adenoc. & $\mathrm{CCC}$ & $\mathrm{CCC}(\mathrm{RD})$ & $\mathrm{HCC}$ & 965 \\
\hline 66. & $\mathrm{~F}$ & Liver & PDA & CUP & $\mathrm{CCC}(\mathrm{RD})$ & Cervix & 1100 \\
\hline 70. & M & Peritoneum & Adenoc. & Stomach & Stomach (CD) & Colon & 842 \\
\hline 74. & M & Leg & Carc. & Adnex tumor & Adnex tumor (RD) & Normal & 1010 \\
\hline 76. & M & Liver & Adenoc. & Lower Gl & Small intestine (RD) & Colon & 912 \\
\hline 77. & M & LN axilla & PDC & CUP & Lung (CD) & Breast & 978 \\
\hline 86. & $\mathrm{~F}$ & LN axilla & Adenoc. & CUP & Lung (RD) & Stomach & 1108 \\
\hline 88. & $\mathrm{~F}$ & Peritoneum & Adenoc. & Ovary & Ovary (RD) & Cervix & 1033 \\
\hline 89. & $\mathrm{~F}$ & Liver & PDA & $\mathrm{CCC}$ & CCC (RD) & $\mathrm{CCC}$ & 916 \\
\hline 90. & $\mathrm{~F}$ & Peritoneum & Adenoc & Ovary & Ovary (RD) & Ovary & 781 \\
\hline 92. & M & Liver & Malignant tumor & Angiosarcoma & Angiosarcoma (RD) & Normal & 1097 \\
\hline 95. & M & Peritoneum & PDC & DSRCT & $\mathrm{DSRCT}(\mathrm{RD})$ & Breast & 1098 \\
\hline \multirow[t]{2}{*}{$71+72$} & M & Bone + Kidney & PDC & Kidney & Kidney (RD) & Kidney & 1096 \\
\hline & & & & & & & 1277 \\
\hline \multirow[t]{2}{*}{$75+87$} & $\mathrm{~F} / 43$ & Liver & PDA & $\mathrm{CCC}$ & $\mathrm{CCC}(\mathrm{RD})$ & $\mathrm{CCC}$ & 925 \\
\hline & & & & & & & 1030 \\
\hline ID & Sex & Biopsy site & Histology & Path Diag. & Stand of Ref & LDA Pred & Outlier score \\
\hline 11. & $F$ & LN neck & PDA & CUP & CUP (SD) & Ovary & 756 \\
\hline 13. & $\mathrm{~F}$ & Peritoneum & PDA & CUP & CUP (NSD) & Pancreas & 1193 \\
\hline 21. & M & LN neck & PDC & CUP & CUP (NSD) & Breast & 1108 \\
\hline 26. & $\mathrm{~F}$ & Skin & PDA & CUP & CUP (NSD) & Breast & 971 \\
\hline 32. & M & LN neck & PDSCC & CUP-SCC & CUP (NSD) & Normal & 926 \\
\hline 33. & M & Skin & PDA & CUP & CUP (NSD) & Colon & 1098 \\
\hline 41. & M & Liver & PDA & Pancreas & CUP (NSD) & Stomach & 1040 \\
\hline 42. & M & Liver & Adenoc. & CUP & CUP (NSD) & Pancreas & 994 \\
\hline 43. & $\mathrm{~F}$ & LN retro & PDA & CUP & CUP (NSD) & Stomach & 797 \\
\hline 45. & M & Liver & PDC & CUP & CUP (NSD) & Colon & 1245 \\
\hline 46. & F & Liver & Adenoc. & CUP & CUP (NSD) & Normal & 1027 \\
\hline 47. & $\mathrm{~F}$ & Liver & Adenoc. & CUP & CUP (SD) & $\mathrm{CCC}$ & 932 \\
\hline 48. & $\mathrm{~F}$ & LN neck & PDC & CUP & CUP (NSD) & Ovary & 1032 \\
\hline
\end{tabular}


Table 1 Prediction results in CUP patients (Continued)

\begin{tabular}{|c|c|c|c|c|c|c|c|}
\hline 54. & $\mathrm{~F}$ & Liver & Adenoc. & CUP & CUP (NSD) & Normal & 1068 \\
\hline 55 & $\mathrm{~F}$ & Liver & Adenoc. & CUP & CUP (NSD) & Normal & 962 \\
\hline 58. & $\mathrm{~F}$ & Liver & PDC & CUP & CUP (SD) & CCC & 995 \\
\hline 61. & M & Liver & Carc. & $\mathrm{HCC}$ & CUP (NSD) & $\mathrm{CCC}$ & 1102 \\
\hline 64. & $\mathrm{~F}$ & LN inguien & PDA & CUP & CUP (SD) & Lung & 1168 \\
\hline 65. & M & LN neck & PDSCC & CUP-SCC & CUP (NSD) & Breast & 929 \\
\hline 73. & M & LN retro & PDC & CUP & CUP (NSD) & Normal & 1020 \\
\hline 78. & $\mathrm{~F}$ & Lung & Adenoc. & Lower Gl & CUP (NSD) & Lung & 1062 \\
\hline 80. & $\mathrm{~F}$ & Liver & Adenoc. & CUP & CUP (SD) & CCC & 1111 \\
\hline 81. & $\mathrm{~F}$ & Liver & PDA & CUP & CUP (NSD) & Breast & 1212 \\
\hline 82. & $\mathrm{~F}$ & Bone & Adenoc. & CUP & CUP (NSD) & CCC & 1209 \\
\hline 83. & $\mathrm{~F}$ & Liver & PDA & CUP & CUP (SD) & CCC & 1061 \\
\hline 91. & $\mathrm{~F}$ & LN axilla & Adenoc. & CUP & CUP (SD) & Lung & 939 \\
\hline 93. & M & Bone & PDSCC & CUP-SCC & CUP (NSD) & Breast & 940 \\
\hline 94. & $\mathrm{~F}$ & Liver & PDA & CUP & CUP (NSD) & Normal & 984 \\
\hline \multirow[t]{2}{*}{ 50. +68} & M & Adr gl & PDC & CUP & CUP (NSD) & Stomach & 978 \\
\hline & & & & & & Pancreas & 1079 \\
\hline
\end{tabular}

A validation of the LDA predicted diagnoses was performed by comparing with a Standard of Reference (SR). SR was established by an experienced pathologist and two experienced oncologists. In addition to the 23 patients where a primary tumor site was identified (Reference Diagnosis (RD)) within the study period, the Standard of Reference reached a Consensus Diagnosis (CD) in 5 patients based on patient demographics, metastatic pattern, results of clinical and laboratory tests, imaging data and pathologic evaluations (Samples labeled in red). In the 29 remaining CUP labeled in blue, the results from gene expression profiling were compared with clinicopathological features and the predictions were categorized as Supportive (SD) or Non-Supportive (NSD). LN: lymph node; $\mathrm{n}$ : neck LN; m: mediastinal LN; a: axilla LN; r: retroperitoneal LN; p: pelvis LN; adr gl: adrenal gland; Adenoc: adenocarcinoma, PDA: poorly differentiated adenocarcinoma; Carc: carcinoma; PDC: poorly differentiated carcinoma; SCC: squamous cell carcinoma; PDSCC: poorly differentiated SCC; CCC: cholangiocarcinoma; HCC: hepatocelluar carcinoma; DSRCT: desmoplastic small round cell tumor.Path Diag: pathological diagnosis; Stand of ref: Standard of reference; LDA pred: Linear discriminant analysis prediction; RD: Reference Diagnosis; CD: Consensus Diagnosis, SD: Supportive Diagnosis; NSD: Non-Supportive Diagnosis.

supposed to be compared to metastases of known origin, we subsequently combined the training and validation sets and generated a second classifier, consisting of 641 probe sets (641 classifier). Furthermore a gender correction by renormalizing the prior class probabilities in the test situation was implemented because we noted that tumors from males incorrectly were classified as ovary, cervical and endometrial cancer. The accuracy in primary tumors, known metastases and normal samples of the 641 classifier was $92 \%, 87 \%$ and $89 \%$, respectively (Additional file 3: Table S2) and this classifier was subsequently used for the prediction of CUP. The principal component analysis is shown in Figure $1 \mathrm{~B}$ and the ten most selective transcripts and their gene ontology for each tumor class are listed in Additional file 4: Figure S2.

To provide a systematic overview of the expression of conventional tumor markers in the CUP samples, we also compiled a list of 45 common histopathological biomarkers and depicted their expression in a two-way hierarchal cluster (Figure 2). Whereas, about $85 \%$ of the primary cancers exhibited a characteristic expression of their individual histomarkers, only 10 of the 28 (35\%) CUP - where a putative primary site was identified and 3 of the 29 (10\%) CUP - where the primary site remained unknown - expressed one or more biomarkers at significant levels. The strongest overlap between histopathological markers and the LDA based CUP classifications was observed for CUP predicited as ovary and colorectal cancers, where 4 and 3 samples expressed WT1 or CEA/CEACAM5, respectively. Moreover, 6 samples were positive for TP63 and 2 samples were positive for surfactant proteins. Finally, one sample was positive for $P A X 2$ in agreement with the LDA prediction as renal carcinoma. Compared to the primary cancers there was a limited concordance between markers within the same tumor category. Only two of the WT1 positive cancers were positive for $C A 125 / M U C 16$, and only 3 of the TP63 positive samples expressed $C K 17$ and $C K 5$, characteristic of squamous carcinoma. If the histological markers were combined and used in an LDA based fashion, the concordance with the 641 signature LDA predictions or Standard of Reference was about 66\% indicating that systematic application of the patomarkers may at least to some extent compensate for the modest predictive power of individual markers.

\section{QDA based outlier analysis}

To determine the similarity between primary cancers, metastases of known origin and CUPs, we employed Quadratic Discriminant Analysis (QDA) to determine 

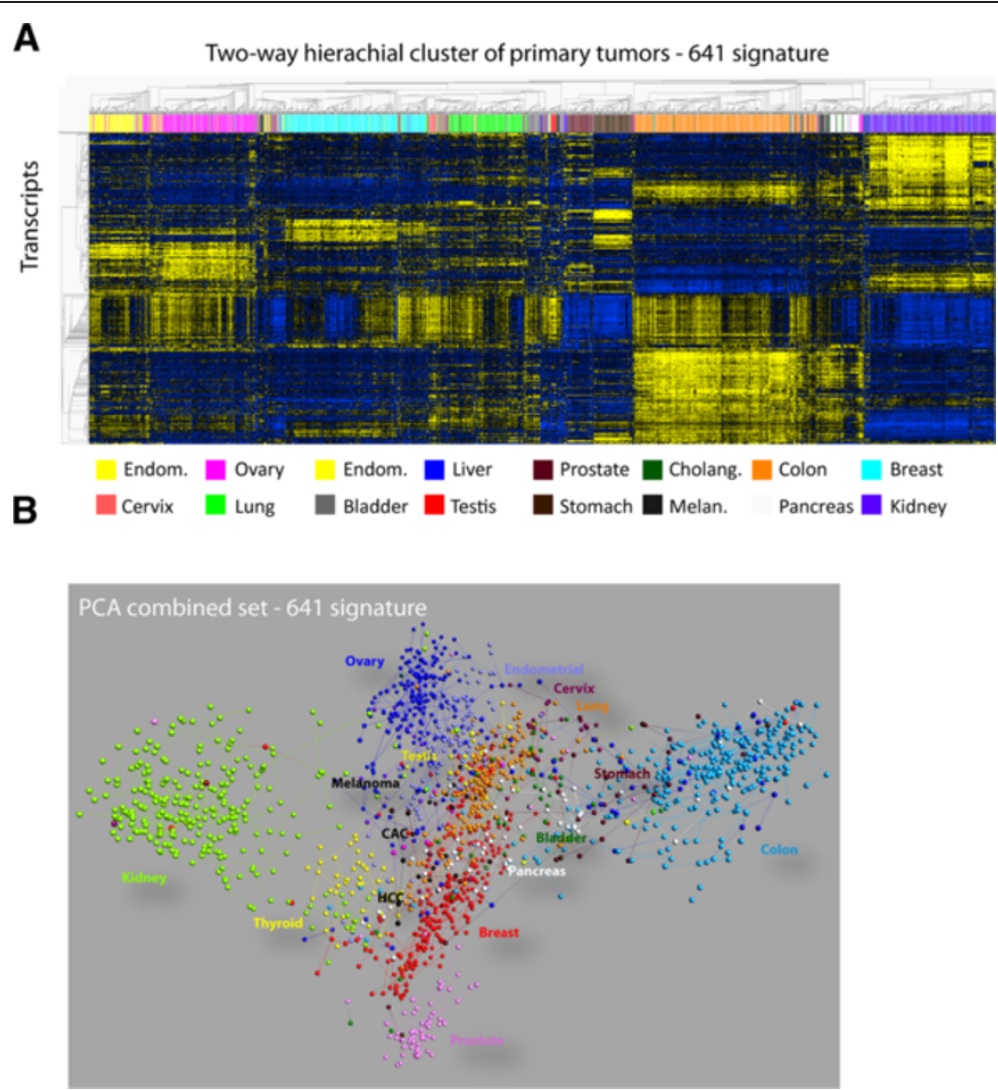

Figure 1 Hierachial cluster and principal component analysis of tumor classes. A. Two-way hierachial cluster of 16 tumor classes by the 641 transcript signature. The tumor classes are shown at the top of the cluster and the transcripts are clustered at the left side. B. Principal component analysis (PCA) of primary tumors and known metastases based on the signature. The tumor classes are colored and indicated in association with the corresponding tumor samples.

the likelihood that a particular sample belonged to one of the predefined tumor classes. Outlier scores were calculated in LOOCV fashion for one sample at a time using all remaining samples i.e. primary tumors and metastases to represent the classes. The outlier scores of the samples from normal tissues are not comparable to the primary tumors and metastases because of the heterogeneity among the many different tissues in the class.

Based on the results from primary tumors and metastases we plotted the predictive error rates versus the outlier scores and demonstrated a clear relationship between errors and outlier scores (Figure 3). Samples with outlier scores below 800 exhibited less than 10\% risk of being erroneous, whereas, outlier scores above 1000 had more than $25 \%$ risk of being incorrect. However, even in the high end of outlier scores with only $75 \%$ accuracy, prediction is far from random, since we are working with 16 different classes. As shown in the box plot (Figure 3), CUP samples had significantly higher outlier values than primary tumors and metastases. To ensure that the difference was not related to our platform, we compared our own samples of known metastases and primary tumors and observed the same difference. CUPs, moreover, consisted of biopsies that may contain more normal tissue than samples obtained during surgery. We therefore plotted the percentage of normal tissue as estimated from the relative expression of markers of lymphoid, liver, and muscle tissue versus the outlier scores, but observed no correlation between the amount of normal tissue in the biopsies and the outlier scores (Additional file 5: Figure S3). A number of samples that expressed conventional histopathological biomarkers exhibited low scores, but if we compared CUPs where a primary cancer was identified during the clinical processing with CUPs where no primary site could be identified, there was no difference between the outlier scores (mean 991 vs mean 1031, P = 0.24). Taken together, the results demonstrate that CUPs are more distantly related to the predefined tumor classes, than known metastases.

\section{mRNA Expression and Gene Set Enrichment in CUP}

To identify differentially expressed transcripts, we performed a class comparison between CUP and metastases of known origin. The analysis was performed as a paired 

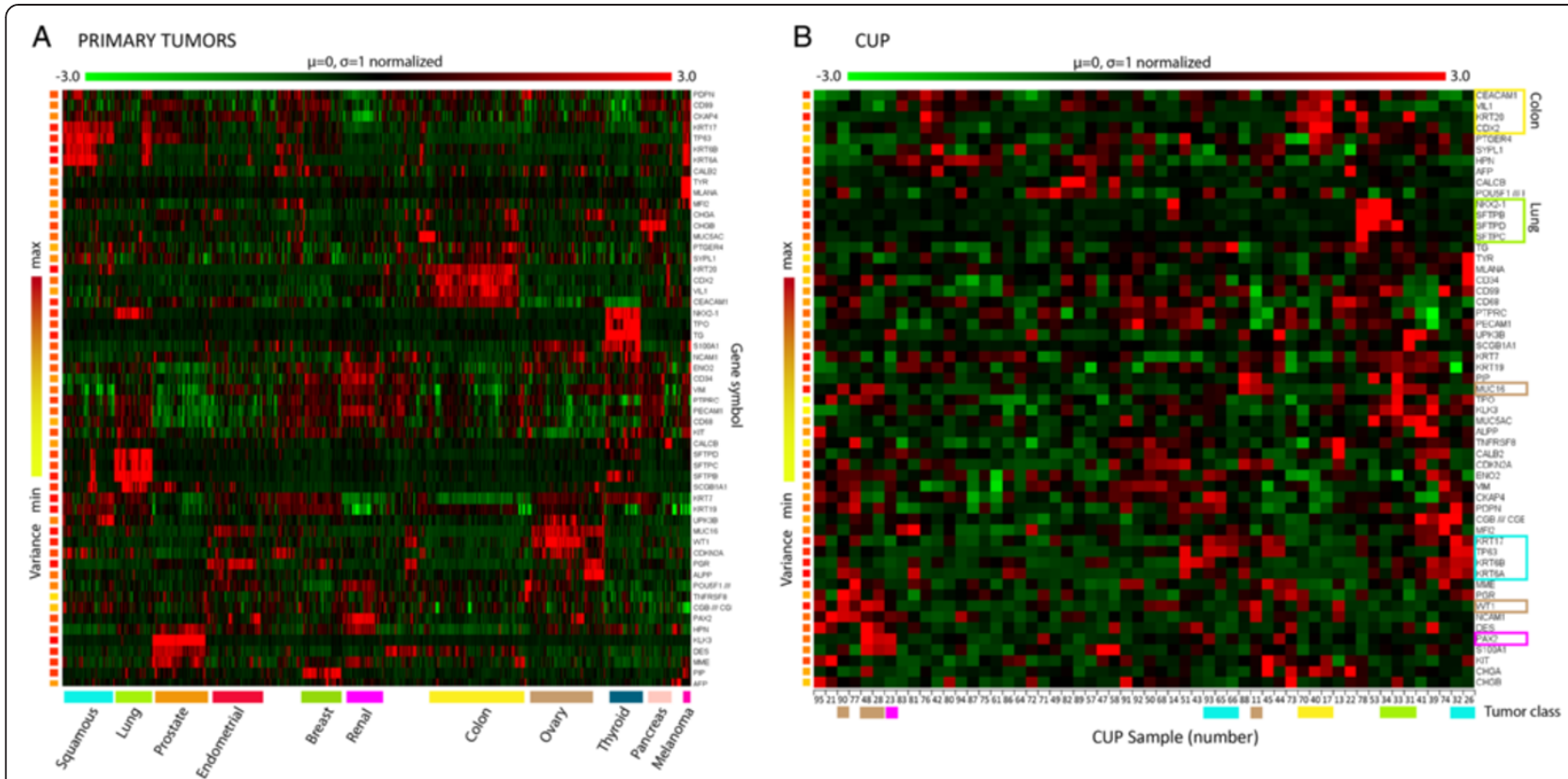

Figure 2 Patomarkers in primary tumors and CUP. Probeset Ids for 45 common histopatological markers were collected and used to generate a two-way hierarchal cluster with a selection of primary tumors (Panel A) or CUP (Panel B). The variance of the individual markers is shown to the left and the scale is indicated at the top of the clusters. Gene symbols are shown to the right and the different tumor classes are shown below ((Panel A), primary tumors). For the CUP samples (Panel B), groups of markers corresponding to different tumor classes are indicated by the boxes around the gene symbols at the right side of the cluster. The number below the cluster indicated the number of the CUP sample corresponding to the annotation in Table 1.
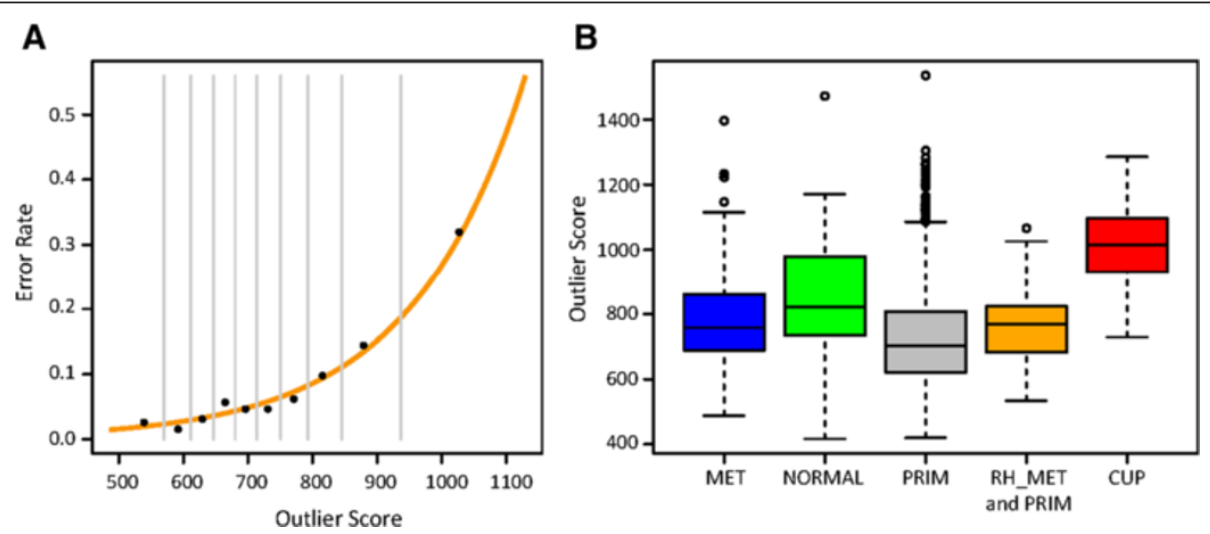

Figure 3 QDA derived outlier scores in CUP. A) To determine the relationship between prediction error and outlier scores the primary cancers and metastases were divided into ten bins according to the outlier scores and the error rate was calculated for each bin. Each point represents the error rate plotted versus the median outlier score of the bin. The vertical lines show the span of outlier scores within the bins. The plot shows that higher outlier score translates into higher error rate. We modeled the relationship between outlier scores and prediction error by fitting polynomial function to the data points (the orange line), and the function allows us to estimate the expected error rate for new samples of unknown origin, once their outlier scores have been determined. B) Samples from CUP patients tend to have higher outlier scores than other cancer patients. The box plot summarizes the distributions of outlier scores within metastases (MET), primary (PRIM) and CUP tumors. There is a clear tendency for CUP samples to have higher outlier score than metastases and primary cancers. The median outlier score of CUP samples of $>1000$ suggests the origin prediction error above $30 \%$. On the other hand, most primary cancers and metastases have outlier scores below 800 , hence the estimated prediction error from 2-10\% (see panel A). Since data for CUP and some primary tumors and metastases were generated at Rigshospitalet, the non-CUP samples from Rigshospitalet are presented as separate group (RH_MET and RH-PRIM), this is to show that the shift in outlier scores was not caused by technical bias. Additionally, the normal, non-cancerous tissue group (NORMAL) is included, and shows the whole range of outlier scores. 
analysis with respect to the LDA predictions to eliminate differences between tumor classes. Metastases from uterine, testis, prostate, melanoma and thyroid cancers were excluded from the analysis because no CUPs had been allocated to these groups. CUPs predicted as normal tissue were also excluded. Moreover, cholangiocarcinomas were omitted from the calculations because they were not represented in the LDA predicted metastases group. In total 41 CUP and 186 metastases comprising 10 different cancer groups were included in the analysis. To define the most up- and down-regulated CUP transcripts, a cut-off of $\mathrm{p}<10^{-8}$ corresponding to a false discovery rate of $\mathrm{q}<$ $1.9^{*} 10^{-7}$ was employed. This resulted in 1550 down- and 1390 up-regulated probe sets corresponding to 1117 and 934 unique annotated genes, respectively. These two lists comprised our CUP core set of differentially expressed transcripts. The 40 most significantly down- or upregulated mRNAs are shown in Additional file 6: Table S4. The lists of genes was subsequently subjected to a Gene Set Enrichment Analysis (GSEA) using the Broad Institute's GSEA database (http://www.broadinstitute.org/gsea/ msigdb). Initially, we searched for enriched gene ontology terms, and this revealed that up-regulated transcripts were associated with GO-terms $(\mathrm{q}<0.01)$ : DNA_INTEGRITY_ CHECKPOINT,DNA_DAMAGE_CHECKPOINT,DNA_ REPLICATION_INITIATION,DNA_PACKAGING, NEGATIVE_REGULATION_OF_DNA_METABOLIC_ PROCESS,CELL_CYCLE_CHECKPOINT;NEGATIVE_ REGULATION_OF_DNA_REPLICATION,CHROMATIN_ REMODELING,DNA_DAMAGE_RESPONSESIGNAL TRANSDUCTION. There were no particular enrichments among the down-regulated mRNAs.

To depict CUP enriched molecular pathways, we further examined if the CUP core set exhibited overlaps with the Molecular Signature Database (MSigDB) curated gene sets. Overlaps between the CUP core set $(\mathrm{p}<$ $10^{-8}$ ) were computed by submission of up- and downregulated probe sets separately (Table 2 ). Gene sets consisting of transcripts that were positively correlated to BRCA1, ATM and CHECK2 expression were highly enriched in the up-regulated CUP core set. The downregulated CUP mRNAs showed fewer significant overlaps but SHEN_SMARCA2_TARGETS_DN gene set, which depict transcripts that are negatively correlated with SMARCA2 expression in prostate cancer was clearly overlapping with the CUP set.

To examine the BRCA1 and SMARCA2 pathway networks defined by the SHEN_SMARCA2_TARGET_DN, SHEN_SMARCA2_TARGET_UP and PUJANA_BRCA1_ PCC_NETWORK in greater detail, we generated two way clusters using the complete gene sets on our CUP core set (Figure 4). The clusters were based on a paired analysis with respect to their LDA predictions and with the same inclusion criteria, as described above. The
SHEN_SMARCA2_TARGET_DN; SHEN_SMARCA2 TARGET_UP and PUJANA_BRCA1_PCC_NETWORK gene symbols were translated into probe sets and to exclude non-functional redundant probe sets, only the probe sets with the $50 \%$ highest variance were included. We moreover applied a p-value cut-off of 0.001 to filter probe sets that differed among the two groups (Figure 4). The PUJANA_BRCA1_PCC_NETWORK set of genes consists of 1671 gene symbols that translated into 3897 probe sets. Following filtering 705 probe sets corresponding to 519 up-regulated and 66 down-regulated genes were clustered (Figure 4). From the cluster it is apparent that the BRCA1 profile is strongly enriched in CUP compared to the corresponding metastases. A schematic representation of the BRCA1 and non-homologous repair networks showing the enriched factors is depicted in Additional file 7: Figure S4. Following the same procedure, we subsequently looked at the SMARCA2 networking (Figure 4). The SHEN sets consist of 360 SMARCA2 negatively- and 430 SMARCA2 positively- correlated genes that translated into 772 and 1211 probe sets respectively. In the SMARC2A negatively correlated group, we observed 20 genes that were up-regulated and 95 that were down-regulated in CUP compared to metastases, and amongst the SMARCA2 positive correlated genes we saw 161 up-regulated genes and 19 down regulated after filtering (top 50\% variance probes and $\mathrm{p}<0.001$ ). Taken together, the GSEA shows that CUPs are characterized by enrichment of the double strand break DNA repair system and the SMARCA2/BRM chromatin dependent remodeling system.

\section{Chromosome instability in CUP}

Since the observed enrichment of genes involved in DNA double-strand break repair (Additional file 7: Figure S4) indicated that CUPs were more chromosome unstable than known metastasis and primary cancers, we examined the status of signatures involved in DNA repair and genome instability. Signatures of chromosomal instability (CIN), DNA double-strand break repair, nucleotide excision repair (NER), base excision repair (BER) and mismatch repair (MMR) were included to obtain a complete overview of DNA- repair and stability in CUP (Figures 5 and 6). The predefined gene sets were examined with the Broad Institute GSEA v2 software. The expression data matrix was preprocessed in Qlucore Omics Explorer $^{\mathrm{rm}}$ and expression values were normalized within LDA predictions - so the expression values became expressed as a relative value compared to the mean expression of a gene within its group. The data set was analyzed against the 10 selected gene sets (Figure 5) employing 1000 permutations with standard GSEA settings. The most significant scores were observed for the signature of double strand break repair and for signatures of unstable sarcoma [9] and CIN 
Table 2 Enriched or depleted gene sets in CUPs compared to metastases of known origin Up-regulated in CUP

Gene Set name
Transcripts Overlap k/K p value

\section{PUJANA_BRCA1_PCC_NERWORK}

Genes constituting the BRCA1-PCC network of transcripts whose expression positively correlated (Pearson correlation coefficient, PCC $>=0.4$ ) with that of BRCA1

1671

KINSEY_TARGETS_OF_EWSR1_FLII_UP

Genes up-regulated in TC71 and EWS502 cells (Ewing's sarcoma) upon knockdown of theEWSR1-FLII fusion PUJANA_ATM_PCC_NETWORK

Genes constituting the ATM-PCC network of transcripts whose expression positively correlated (Pearson correlation coefficient, PCC $>=0.4$ ) with that of ATM

\section{PUJANA_CHEK2_PCC_NETWORK}

Genes constituting the CHEK2-PCC network of transcripts whose expression positively correlates (Pearson correlation coefficient, PCC > 0.4) with that of CHEK2

\section{DODD_NASOPHARYNGEAL_CARCINOMA_DN}

Genes down-regulated in nasopharyngeal carcinoma (NPC) compared to the normal tissue.

RODRIGUES_THYROID_CARCINOMA_ANAPLASTIC_UP

Genes up-regulated in anaplastic thyroid carcinoma (ATC) compared to normal tissue.

MILI_PSEUDOPODIA_HAPTOTAXIS_UP

Transcripts enriched in pseudopodia of $\mathrm{NIH} / 3 \mathrm{~T} 3$ cells (fibroblast) in response to haptotactic migratory stimulus by fibronectin, FN1

\section{RODRIGUES_THYROID_CARCINOMA_POORLY_DIFFERENTIATED_UP}

Genes up-regulated in poorly diffrentiated thyroid carcinoma (PDTC) compared to normal thyroid tissue.

DECOSTA_UV_RESPONSE_VIA_ERCC3_DN

Genes down-regulated transcripts in fibrolasts expressing ethier XP/CS or TDD mutant forms of ERCC3 855 [Gene ID=2071], after UVC irradiation

DECOSTA_UV_RESPONSE_VIA_ERCC3_COMMON_DN

Common down -regulated transcripts in fibroblasts expressing either XP/CS orTDD mutant forms of ERCC3 [Gene ID=2071], after UVC irradiation

\section{OSMAN_BLADDER_CANCER_UP}

Common down-regulated in blood samples from bladder cancer patients

SENUPTA_NASOPHARYNGEAL_CARCINOMA_WITH_LMP1_UP

Genes up-regulated in nasopharyngeal carcinoma (NPC) positive for LMP1 [Gene ID=9260], a latent gene of Epstein Barr virus (EBV)

\section{SENUPTA_NASOPHARYNGEAL_CARCINOMA_UP}

Genes up-regulated in nsopharyngeal carcinoma relative to the normal tissue.

\section{PUJANA_XPRSS_INT_NETWORK}

Genes constituting the XPRSS-Int network: intersection of genes whose expression correlates with BRCA1, BRCA2, ATM, and CHEK2 [Gene ID=672, 675, 472, 11200] in a compendium of normal tissues.

$\begin{array}{llll}1281 & 133 & 0.1038 & 0.00 E+00 \\ 1461 & 152 & 0.104 & 0.00 E+00\end{array}$

782

$89 \quad 0.1138 \quad 0.00 E+00$

$\begin{array}{llll}1375 & 157 & 0.1142 & 0.00 \mathrm{E}+00\end{array}$

$\begin{array}{llll}721 & 93 & 0.129 & 0.00+00\end{array}$

$\begin{array}{llll}552 & 74 & 0.1341 & 0.00 E+00\end{array}$

$\begin{array}{llll}640 & 94 & 0.1469 & 0.00 \mathrm{E}+00\end{array}$

$126 \quad 0.1474 \quad 0.00 \mathrm{E}+00$

$\begin{array}{llll}420 & 64 & 0.1524 & 0.00 E+00\end{array}$

$402 \quad 57 \quad 0.1418 \quad 5.55 E-16$

$399 \quad 56 \quad 0.1404 \quad 155 E-15$

$286 \quad 46 \quad 0.1608 \quad 3.33 E+15$

$\begin{array}{llll}167 & 34 & 0.2036 & 1.21 \mathrm{E}-14\end{array}$

\begin{tabular}{|c|c|c|c|c|}
\hline \multicolumn{5}{|l|}{ Down-regulated in CUP } \\
\hline Gene Set Name & Transcripts & Overlap & $\mathbf{k} / \mathrm{K}$ & p value \\
\hline \multicolumn{5}{|l|}{ SHEN_SMARCA2_TARGETS_DN } \\
\hline $\begin{array}{l}\text { Genes whose expression negatively correlated with that of SMARCA2 [GenelD=6595] in prostate cancer } \\
\text { samples }\end{array}$ & 360 & 73 & 032028 & $0.00 E+00$ \\
\hline \multicolumn{5}{|l|}{ GINESTIER_BREAST_CANCER_ZNF217_AMPLIFIED_DN } \\
\hline $\begin{array}{l}\text { Genes doen-regulated in non-metastic breast cancer tumors having type } 1 \text { amplifications in the } 20 q 13 \\
\text { region; involves ZNF217 [Gene ID=7764] locus only. }\end{array}$ & 336 & 49 & 0.1458 & $7.71 \mathrm{E}-11$ \\
\hline
\end{tabular}
region; involves ZNF217 [Gene ID=7764] locus only.

Gene set enrichments among up or down regulated mRNAs in the CUP core set were examined in the molecular signatures database (MSig) among the C2 curated gene sets comprising profiles from chemical and genetic perturbations, canonical pathways, BIOCARTA, KEGG and the reactome collections. The uncorrected $\mathrm{p}$ values are indicated. In all cases the false positive discovery rate was set to $q<0.01$. 

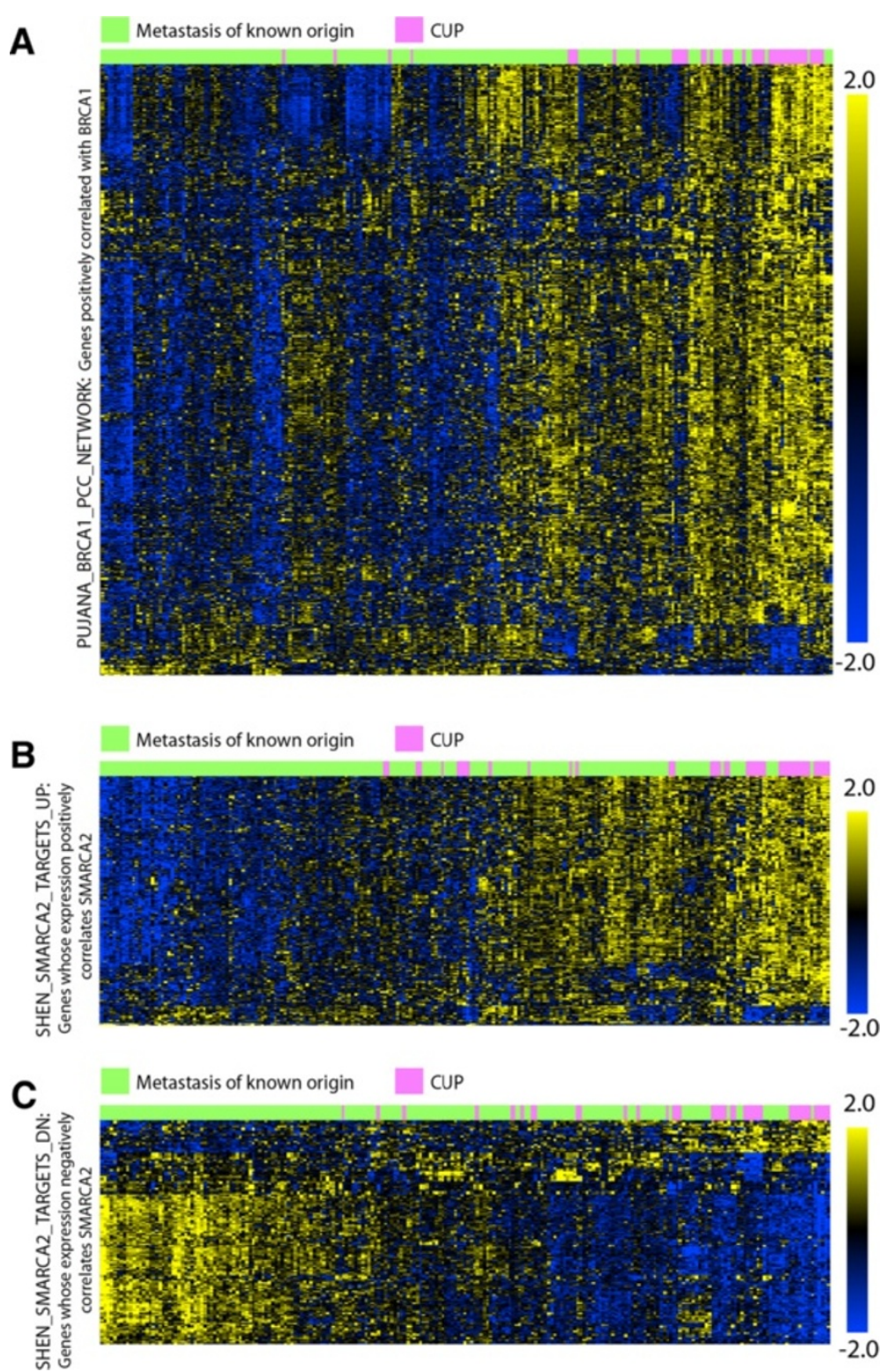

Figure 4 Two way hierachial clusters of BRCA1 and SMARCA2 networks in metastases and CUP. (A) The PUJANA_BRCA1_PCC_NETWORK was downloaded from the MSig database (http://www.broadinstitute.org/gsea/msigdb) and used to generate a paired two way hierarchical cluster with known metastases and CUPs. Gene symbols were translated into probe sets and because of the probe set redundancy the data were filtered by a $p<0.001$ before clustering. Following filtering 1297 probe sets were included in the clustering. Known metastases are indicated in green and CUP samples are labeled with pink above the cluster. The scale is shown at the right side of the cluster. (B) Two-way cluster of the SHEN_SMARCA2_TARGETS up- and (C) downregulated transcripts. The sets consists of 360 down- and 430 up-regulated genes that translated into 772 and 1211 probe sets, respectively. The known metastases are indicated in pink and CUP samples are labeled with green below the cluster. The scale is shown at the right side of the cluster.

[10]. Moreover, the KEGG signature of NER was enriched but not to a significant level $(\mathrm{p}=0.123)$. The remaining nucleotide excision and mismatch repair signatures were not enriched in CUP and we infer that CUPs primarily distinguishes themselves from metastasis of known origin by signatures of chromosome instability. The signature of chromosome unstable sarcoma was finally employed to generate an instability score providing an index of the chromosomal instability for comparison of normal tissue, primary cancers and metastasis and CUP (Figure 6). The instability score was calculated as the mean of the expression values from the included probe sets of the signature following variance filtering (206 probe sets). As shown in Figure 6 panel B CUP exhibited a significantly higher score than paired metastasis of known origin. Metastases were significantly more chromosomal unstable than primary 


\begin{tabular}{|c|c|c|c|}
\hline MsigDB Geneset & Size & NES & Transcript Ranking \\
\hline REACTOME_DOUBLE_STRAND_BREAK_REPAIR & 21 & 1.85 & \\
\hline FERREIRA_EWINGS_SARCOMA_UNSTABLE_VS_STABLE_UP & 129 & 1.70 & \\
\hline CARTER_ZSALLASI_CIN_70 & 70 & 1.53 & \\
\hline KEGG_NUCLEOTIDE_EXCISION_REPAIR & 44 & 1.52 & \\
\hline REACTOME_GLOBAL_GENOMIC_NER & 33 & 1.48 & \\
\hline REACTOME_NUCLEOTIDE_EXCISION_REPAIR & 49 & 1.39 & \\
\hline REACTOME_TRANSCRIPTION_COUPLED_NER & 44 & 1.27 & \\
\hline KEGG_BASE_EXCISION_REPAIR & 33 & 1.27 & \\
\hline REACTOME_BASE_EXCISION_REPAIR & 17 & 1.19 & \\
\hline WATANABE_COLON_CANCER_MSI_VS_MSS_UP & 28 & 1.16 & \\
\hline
\end{tabular}

Figure 5 Signatures of genomic instability in CUPs. Messenger RNA signatures of chromosomal instability (CIN), DNA double-strand break repair, nucleotide excision repair (NER), base excision repair (BER) and mismatch repair (MMR) in CUPs and MOKO were examined with the Broad Institute GSEA v 2 software. The names of the individual signatures, the number of transcripts and the normalized enrichment scores (NES) are indicated. The right panel depicts the transcript ranking on a colometric scale. With the exception of the CIN signature obtained from [10] all gene lists were retrieved from http://www.broadinstitute.org/gsea/msigdb.

cancers and as expected normal tissues exhibited very low instability scores. We moreover examined the correlation between outlier scores and instability scores, because chromosome instability is envisioned to promote evolution and phenotypic variations. In a linear correlation analysis including primary cancers, metastasis and CUPs the outlier scores were positively correlated with the chromosomal instability score $(\mathrm{p}<7.24 \mathrm{E}-33 ; \mathrm{q}<2.16 \mathrm{E}-30)$, so we infer that chromosomal instability is likely to be implicated in the phenotypic traits of CUPs.

\section{Discussion}

To examine the biological differences between metastasis of known origin and CUPs, we first developed a robust LDA classifier that could define the most likely origin of a particular CUP on a molecular basis. In line with a number of previous molecular prediction studies [11-25] CUPs were predicted to mainly emanate from bile duct/cholangiocarcinoma, breast, lung and colorectal cancers (Additional file 8: Table S3). Quadratic discrimant analysis (QDA) was subsequently used to calculate the distance of primary tumors, metastases and our CUP samples to the nearest tumor class. In agreement with the acquisition or loss of phenotypic traits compared to their origin, CUPs were more distantly related to the predefined tumor classes than known metastases.

The explanation for the disparity between CUPs and known metastases could obviously be that CUPs were derived from types or subclasses of cancers not represented among our 16 classes, but a number of arguments speak against this. Firstly, autopsy and previous molecular classification studies support that the vast majority of CUPs are likely to originate from the included tumor classes [26]. Secondly, the genetic signature was selected by means of an F-test considering the entire class, so class specific transcripts are supposed to be present even in putative subclasses. Thirdly, high outlier scores were also observed among classes such as colorectal cancers that are not known to contain subclasses. Finally, if a number of CUPs represented rare cancers, the majority of the CUP scores should have overlapped with metastases of known origin. So, taken together, we infer that the observed difference in outlier scores is likely to reflect that CUPs exhibit distinct molecular features.

Attempts to elucidate the molecular biology of CUPs have been hampered by the heterogeneity of the cancers and their elusive origins. Previous studies have indicated that activation of MAPK, cMET and pAKT axes were associated with progression and outcome in CUPs, whereas p21 expression conferred a favorable prognosis [27-29]. Based on the LDA predictions it became, however, possible to define CUP enriched transcripts and molecular pathways in a paired analysis with metastasis of known origin. At the single transcript level one of the most consistently down-regulated factors was early growth response 1 (EGR1), which is involved in cell growth and differentiation. Suppressed EGR1 levels have previously been reported in breast carcinoma [30], glioblastoma [31] and lung [32] cancer, where it was predictive of poor outcome.

The search for gene set enrichments moreover showed that CUPs were enriched in transcripts encoding BRCA1, ATM and CHEK2 DNA damage and homologous 
A Ferriera_unstable_UP(149 genes, 309 probesets) p<0.001 Genes: (84 up 23 down)

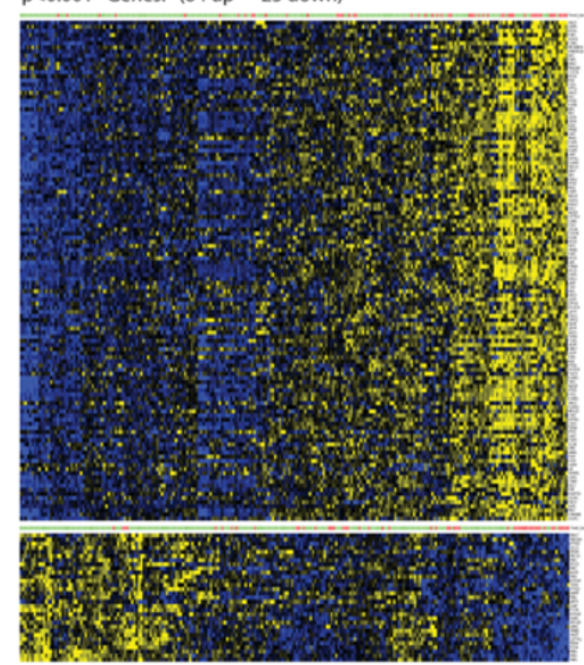

C

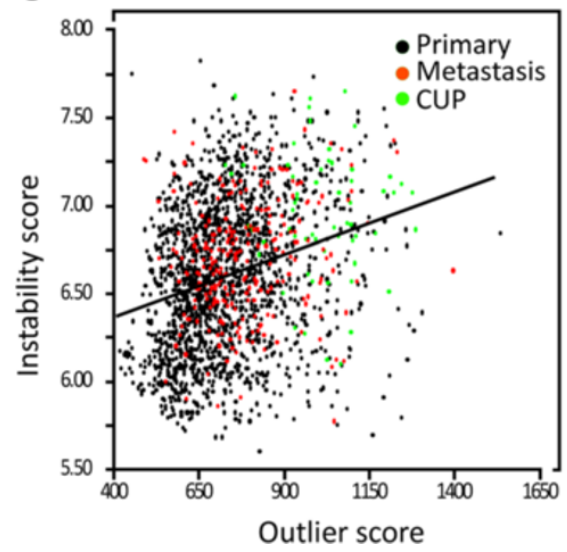

B

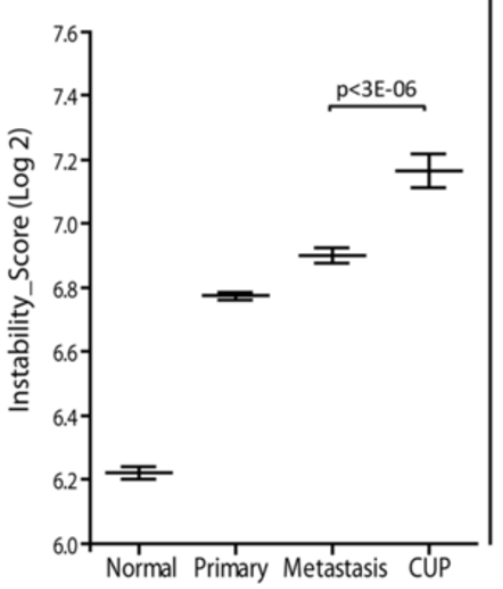

Linear Correlation: Outlier score vs Instability score $\mathrm{p}<1.14 \mathrm{E}-33$

Figure 6 Chromosomal instability and outlier scores in CUP. Panel A. Two-way hierachial cluster of the Ferreira_Ewing_Sarcoma_Unstable signature in MOKO and CUPS. MOKO and CUPs are indicated by green and pink labels, respectively. Panel B. Instability scores in Normal tissues, Primary tumors, MOKO and CUPs. The signature of chromosome unstable sarcoma was employed to generate an instability score calculated as the mean of the expression values from the included probe sets of the signature following variance filtering (206 probe sets). Panel C. Linear correlation between outlier scores and instability scores. Primary tumors, metastasis of known origin and CUP are indicated as black, red or green dots, respectively. The lower panel shows the value of the instability scores depicted in a green to red color scale. The p-value of the linear correlation between outlier and instability scores is indicated.

recombination repair networks suggesting that CUP are chromosome unstable. This was corroborated by the direct demonstration of mRNA signatures of chromosome instability (CIN) in CUPs. CIN is strongly associated with poor outcome and drug resistance [10], and CIN positive tumors reconcile many of the characteristics of CUP (for review [33]). Previous analyses of the cytogenetic profile of CUPs have also shown that chromosomal changes are frequent and widespread in CUP and that CUPs exhibiting large numbers of chromosomal aberrations have a poor outcome [34]. CUPs are moreover frequently resistant to platinum salts (for review [35] in agreement with the presence of CIN and concurrent up-regulation of the homologous recombination repair [36].

The molecular basis of CIN in sporadic cancers is incompletely understood but oncogene induced collapse of DNA replication forks, leading to DNA double strand breaks and genomic instability is considered an appealing model [37]. CIN is a major driving force for tumorigenesis because it promotes accumulation of transforming genotypes and increases the acquisition of independent phenotypic traits, that may translate into atypical presentations 
[38]. In agreement with this we observed a clear correlation between the CIN score and the outlier scores that portrays the similarity of the cancers to known groups.

Systemic cancer progression has been proposed to occur via two models. The prevailing model states that cancer progression occurs within the primary tumor before metastatic dissemination of fully malignant cells, whereas the second brings forward that cells disseminate from the primary tumor at an early stage and pursues a parallel and independent progression of metastases (reviewed in [39]). The two models provide a rationale for the observed difference between metastases of known origin and CUPs, because the parallel progression predicts greater disparity between metastatic founders and primary tumor cells than does linear progression. By inference, CIN is likely to facilitate parallel progression by the early accumulation of distinct genetic and epigenetic alterations in the primary tumor and metastases. Moreover, tumor cells are predicted to settle at unconventional sites due to their independent selection and spread before the primary cancer causes clinical symptoms.

\section{Conclusion}

We conclude that CUPs are characterized by chromosome instability, which distinguishes them from metastases of known origin. We propose that CIN and parallel metastatic behavior may be implicated in early dissemination and poor outcome of CUPs.

\section{Availability of supporting data}

Gene expression CEL files are available at Gene Expression Omnibus (GEO) (http://www.ncbi.nlm.nih.gov/geo/) (Testset: GSE2109, GSE7307, GSE6004, GSE6764, GSE10135, GSE2328, GSE13471, GSE7392 and GSE12606) (Validationset: GSE2109, GSE3325, GSE5764, GSE5764, GSE5787, GSE7307, GSE7476, GSE7553, GSE10245, GSE11151, GSE14762, GSE15471, GSE17537, GSE19826, GSE19829, GSE20565) or at ArrayExpress https://www.ebi.ac.uk/ arrayexpress/ accession E-MTAB-3222 (samples collected and processed at our own facility). Thyroid samples can be retrieved from accession E-MEXP-2442. The specific identifiers are depicted in Additional file 1: Table S1. Supplemental scripts for the outlier analysis and classifier are available at: http://www.genomic-medicine.dk/ recent-papers-and-additional-f/supplemental-files-forrecent-publications/.

\section{Additional files}

Additional file 1: Classification and outlier scores of primary tumors, metastasis and CUP.

Additional file 2: Figure S1. CUP patients and the processing of tumor samples. Sixty-eight CUP patients were consecutively enrolled in the study. In 60 patients the biopsy was obtained during the diagnostic work-up, whereas eight patients received first-line CUP therapy prior to the biopsy. Eleven tumor samples were excluded because the RNA integrity or yield did not meet the required quality criteria. In this way the number of CUP patients ended up at 57. During the diagnostic work a primary tumor site was identified in 23 patients (Table 1) and a consensus diagnosis based on patient demographics, metastatic pattern, results of clinical and laboratory tests, imaging data and pathological evaluations was obtained in five patients as described. In 29 patients the primary tumor site remained unknown.

Additional file 3: Table S2. Prediction of tumor classes with the 428 and 641 classifiers.

Additional file 4: Figure S2. Gene families and tumormarkers A. The 10 most selective transcripts in the 641 classifier for each tumor class. Transcripts were selected by comparison of the indicated tumor class with all other primary cancers and normal tissues and the ten transcripts exhibiting the lowest $p$ value are shown. Due to the small sample number in cholangiocarcinoma and hepatocellular carcinoma, transcripts exhibited $p<0.01$ and $10^{-4}$, respectively. For all other groups $p<10^{-10}$. Genes marked in red were considered to be almost exclusive to the class. B. Comparison of gene families in the patomarker and 641 classifier gene sets. Gene symbols of the two gene sets were submitted to the Brookhaven gene set enrichment analysis molecular signatures database (http://www.broadinstitute.org/gsea/ msigdb) and examined with the Gene Families feature. The diagram shows the percentage of transcripts in each functional category. The 641 classifier transcripts are shown in red bars and the patomarkers are shown in blue bars.

Additional file 5: Figure S3. Percentage of normal cells in CUP biopsies and QDA derived outlier scores. The percentage of normal cells in the CUP biopsies was determined by comparing the levels of liver (APOA2, ALB), muscle (ACTA1), lymph node (IGJ, IGHA1, IGKV3-20) and skin (KRT2, TYRP1) specific transcripts in the samples with the expression of the transcripts in corresponding normal tissues where the biopsies were obtained. The number indicates the CUP sample code and samples labeled in yellow were classified as normal tissue. The blue graph shows the corresponding outlier score generated by the QDA analysis.

Additional file 6: Table S4. Forty most up--(red) or down--regulated (blue) mRNAs in CUP compared to metastasis of known origin. Differentially expressed transcripts, were retrieved by a class comparison between CUPS and metastasis The analysis was performed as a paired analysis with respect to the LDA predictions to eliminate differences related to the individual tumor classes. Metastases from endometrial, testis, prostate, melanoma and thyroid cancers were excluded from the analysis because no CUP had been allocated to these groups by the LDA. CUPs predicted as normal tissue were also excluded.

Additional file 7: Figure S4. Schematic representation of CUP enriched BRCA1 DNA damage response and non-homologous end joining repair networks. Diagrams were generated by the Ingenuity software (Ingenuity systems, USA). Up-regulated factors are indicated in red.

Additional file 8: Table S3. LDA predictions in CUP.

Competing interests

The authors declare that they have no competing interests.

\section{Authors' contributions}

GD and FCN conceived the study, participated in its design and coordination and wrote the manuscript. FJ performed the ultrasound guided biopsy of CUP patients. AKHM, RB and KP collected, organized and annotated the gene expression library. BK, RH, RB, OW and AK created the CUP classifier and the calculations of the outlier score. JV and FCN performed all the qlucore based array data analysis including GSEA and made the biological interpretations of the results. All authors read and approved the final manuscript.

\section{Acknowledgments}

Elisabeth Schiefloe and Susanne Smed are thanked for their technical assistance and Leila Majdanac for secretarial assistance and proof-reading. This study was supported by grants from the Danish National Advanced Technology Foundation, the Danish Cancer Society, the Svend Andersen Foundation, the Toyota Foundation and the NOVO-Nordisk Foundation. 


\section{Author details}

${ }^{1}$ Center for Genomic Medicine, Rigshospitalet, University of Copenhagen, Blegdamsvej 9, DK-2100 Copenhagen $\varnothing$, Denmark. ²Department of Oncology, University of Copenhagen, Blegdamsvej 9, DK-2100 Copenhagen $\varnothing$, Denmark. ${ }^{3}$ Bioinformatics Centre, Department of Biology and Biotech Research and Innovation Centre, University of Copenhagen, DK-2200 Copenhagen, Denmark. ${ }^{4}$ Department of Radiology Copenhagen University Hospital, Rigshospitalet, Blegdamsvej 9, DK-2100 Copenhagen Ø, Denmark. ${ }^{5}$ Technical University of Denmark (DTU), DK-2800 Lyngby, Denmark.

Received: 19 August 2014 Accepted: 24 February 2015 Published online: 19 March 2015

\section{References}

1. Pavlidis N, Briasoulis E, Hainsworth J, Greco FA. Diagnostic and therapeutic management of cancer of an unknown primary. Eur J Cancer. 2003;39:1990-2005.

2. Pavlidis N, Fizazi K. Carcinoma of unknown primary (CUP). Crit Rev Oncol Hematol. 2009;69:271-8.

3. Daugaard G, Møller A, Petersen B. Carcinoma of Unknown Primary. In: Cavalli HH F, Kaye S, Amritage J, Pickard M, editors. Textbook of Medical Oncology. 4th ed. 2009. p. 313-22.

4. Stella GM, Senetta R, Cassenti A, Ronco M, Cassoni P. Cancers of unknown primary origin: current perspectives and future therapeutic strategies. J Transl Med. 2012;10:12

5. Wicha MS, Hayes DF. Circulating tumor cells: not all detected cells are bad and not all bad cells are detected. J Clin Oncol. 2011;29(12):1508-11.

6. Briasoulis E, Tolis C, Bergh J, Pavlidis N. ESMO Minimum Clinical Recommendations for diagnosis, treatment and follow-up of cancers of unknown primary site (CUP). Ann Oncol. 2005;16:75-6.

7. Borup $\mathrm{R}$, Rossing $M$, Henao $\mathrm{R}$, et al. Molecular signatures of thyroid follicular neoplasia. Endocr Relat Cancer. 2010;17(3):691-708.

8. Bolstad BM, Irizarry RA, Astrand M, Speed TP. A comparison of normalization methods for high density oligonucleotide array data based on variance and bias. Bioinformatics. 2003;19:185-93.

9. Ferreira BI, Alonso J, Carrillo J, Acquadro F, Largo C, Suela J, et al. Array CGH and gene-expression profiling reveals distinct genomic instability patterns associated with DNA repair and cell-cycle checkpoint pathways in Ewing's sarcoma. Oncogene. 2008;27:2084-90.

10. Carter SL, Eklund AC, Kohane IS, Harris LN, Szallasi Z. A signature of chromosomal instability inferred from gene expression profiles predicts clinical outcome in multiple human cancers. Nat Genet. 2006;38:1043-8.

11. Talantov D, Baden J, Jatkoe T, Hahn K, Yu J, Rajpurohit Y, et al. A quantitative reverse transcriptase-polymerase chain reaction assay to identify metastatic carcinoma tissue of origin. J Mol Diagn. 2006:8:320-9.

12. Tothill RW, Kowalczyk A, Rischin D, Bousioutas A, Haviv I, van Laar RK, et al. An expression-based site of origin diagnostic method designed for clinical application to cancer of unknown origin. Cancer Res. 2005;65:4031-40.

13. Ma XJ, Patel R, Wang X, Salunga $R$, Murage J, Desai $R$, et al. Molecular classification of human cancers using a 92-gene real-time quantitative polymerase chain reaction assay. Arch Pathol Lab Med. 2006;130:465-73.

14. Su Al, Welsh JB, Sapinoso LM, Kern SG, Dimitrov P, Lapp H, et al. Molecular classification of human carcinomas by use of gene expression signatures. Cancer Res. 2001:61:7388-93.

15. Bloom G, Yang IV, Boulware D, Kwong KY, Coppola D, Eschrich S, et al. Multi-platform, multi-site, microarray-based human tumor classification. Am J Pathol. 2004;164:9-16.

16. Al-Brahim N, Ross C, Carter B, Chorneyko K. The value of postmortem examination in cases of metastasis of unknown origin-20-year retrospective data from a tertiary care center. Ann Diagn Pathol. 2005;9:77-80.

17. Varadhachary $G R$, Spector $Y$, Abbruzzese $J L$, et al. Prospective gene signature study using microRNA to identify the tissue of origin in patients with carcinoma of unknown primary. Clin Cancer Res. 2011;17(12):4063-70.

18. van Laar RK, Ma XJ, de Jong D, Wehkamp D, Floore AN, Warmoes MO, et al. Implementation of a novel microarray-based diagnostic test for cancer of unknown primary. Int J Cancer. 2009;125:1390-7.

19. Varadhachary GR, Talantov D, Raber MN, Meng C, Hess KR, Jatkoe $T$, et al. Molecular profiling of carcinoma of unknown primary and correlation with clinical evaluation. J Clin Oncol. 2008;26:4442-8.

20. Morawietz L, Floore A, Stork-Sloots L, et al. Comparison of histopathological and gene expression-based typing of cancer of unknown primary. Virchows Arch. 2010;456(1):23-9.
21. Monzon FA, Medeiros F, Lyons-Weiler M, et al. Identification of tissue of origin in carcinoma of unknown primary with a microarray-based gene expression test. Diagn Pathol. 2010;5:3.

22. Horlings HM, van Laar RK, Kerst JM, Helgason HH, Wesseling J, van der Hoeven JJ, et al. Gene expression profiling to identify the histogenetic origin of metastatic adenocarcinomas of unknown primary. J Clin Oncol. 2008;26:4435-41.

23. Greco FA, Spigel DR, Yardley DA, et al. Molecular profiling in unknown primary cancer: accuracy of tissue of origin prediction. Oncologist. 2010;15(5):500-6.

24. Hainsworth JD, Henner WD, Pillai R, and Greco FA. Molecular tumor profiling in the diagnosis of patients with carcinoma of unknown primary (CUP): Retrospective evaluation of the Tissue of Origin Test (Pathwork Diagnostics). J Clini Oncol 2010

25. Bridgewater J, van Laar R, Floore A, Van TVL. Gene expression profiling may improve diagnosis in patients with carcinoma of unknown primary. Br J Cancer. 2008:98:1425-30.

26. Pentheroudakis G, Golfinopoulos V, Pavlidis N. Switching benchmarks in cancer of unknown primary: from autopsy to microarray. Eur J Cancer. 2007:43:2026-36.

27. Golfinopoulos V, Pentheroudakis G, Goussia A, Siozopoulou V, Bobos M, Krikelis D, et al. Intracellular signalling via the AKT axis and downstream effectors is active and prognostically significant in cancer of unknown primary (CUP): a study of 100 CUP cases. Ann Oncol. 2012;23:2725-30.

28. Krikelis D, Pentheroudakis G, Goussia A, Siozopoulou V, Bobos M, Petrakis D, et al. Profiling immunohistochemical expression of NOTCH1-3, JAGGED1, CMET, and phospho-MAPK in 100 carcinomas of unknown primary. Clin Exp Metastasis. 2012;29:603-14.

29. Stella GM, Benvenuti S, Gramaglia D, Scarpa A, Tomezzoli A, Cassoni P, et al. MET mutations in cancers of unknown primary origin (CUPS). Hum Mutat. 2011:32:44-50

30. Huang RP, Fan Y, de Belle I, Niemeyer C, Gottardis MM, Mercola D, et al. Decreased Egr-1 expression in human, mouse and rat mammary cells and tissues correlates with tumor formation. Int J Cancer. 1997;72:102-9.

31. Calogero A, Arcella A, De Gregorio G, Porcellini A, Mercola D, Liu C, et al. The early growth response gene EGR-1 behaves as a suppressor gene that is down-regulated independent of ARF/Mdm2 but not p53 alterations in fresh human gliomas. Clin Cancer Res. 2001;7:2788-96.

32. Levin WJ, Press MF, Gaynor RB, Sukhatme VP, Boone TC, Reissmann PT, et al. Expression patterns of immediate early transcription factors in human non-small cell lung cancer. The Lung Cancer Study Group. Oncogene. 1995:11:1261-9.

33. McGranahan N, Burrell RA, Endesfelder D, Novelli MR, Swanton C. Cancer chromosomal instability: therapeutic and diagnostic challenges. EMBO Rep. 2012;13:528-38.

34. Pantou D, Tsarouha H, Papadopoulou A, Mahaira L, Kyriazoglou I, Apostolikas N, et al. Cytogenetic profile of unknown primary tumors: clues for their pathogenesis and clinical management. Neoplasia. 2003:5:23-31.

35. Lord CJ, Ashworth A. The DNA damage response and cancer therapy. Nature. 2012;481:287-94

36. Greco FA, Burris 3rd HA, Litchy S, Barton JH, Bradof JE, Richards P, et al. Gemcitabine, carboplatin, and paclitaxel for patients with carcinoma of unknown primary site: a Minnie Pearl Cancer Research Network study. J Clin Oncol. 2002;20:1651-6.

37. Negrini S, Gorgoulis VG, Halazonetis TD. Genomic instability-an evolving hallmark of cancer. Nat Rev Mol Cell Biol. 2010;11:220-8.

38. Habermann JK, Bundgen NK, Gemoll T, Hautaniemi S, Lundgren C, Wangsa $D$, et al. Genomic instability influences the transcriptome and proteome in endometrial cancer subtypes. Mol Cancer. 2011;10:132.

39. Klein CA. Parallel progression of primary tumours and metastases. Nat Rev Cancer. 2009:9:302-12 